\title{
The Effect of OPC Factor ${ }^{\mathrm{TM}}$ on Energy Levels in Healthy Adults Ages 45-65: A Phase Ilb Randomized Controlled Trial
}

\author{
Patrick J. LaRiccia, M.D., ${ }^{1}$ John T. Farrar, M.D., Ph.D., ${ }^{2}$ Mary D. Sammel, Sc.D., ${ }^{3}$ \\ and Joseph J. Gallo, M.D., M.P.H. ${ }^{4}$
}

\begin{abstract}
Objective: To determine the efficacy of the food supplement OPC Factor ${ }^{\mathrm{TM}}$ to increase energy levels in healthy adults aged 45 to 65 .

Design: Randomized, placebo-controlled, triple-blind crossover study.

Subjects: Twenty-five (25) healthy adults recruited from the University of Pennsylvania Health System.

Interventions: OPC Factor, ${ }^{\mathrm{TM}}$ (AlivenLabs, Lebanon, $\mathrm{TN}$ ) a food supplement that contains oligomeric proanthocyanidins from grape seeds and pine bark along with other nutrient supplements including vitamins and minerals, was in the form of an effervescent powder. The placebo was similar in appearance and taste.

Outcome measures: Five outcome measurements were performed: (1) Energy subscale scores of the Activation-Deactivation Adjective Check List (AD ACL); (2) One (1) global question of percent energy change (Global Energy Percent Change); (3) One (1) global question of energy change measured on a Likert scale (Global Energy Scale Change); 4. One (1) global question of percent overall status change (Global Overall Status Percent Change); and (5) One (1) global question of overall status change measured on a Likert scale (Global Overall Status Scale Change).

Results: There were no carryover/period effects in the groups randomized to Placebo/Active Product sequence versus Active Product/Placebo sequence. Examination of the AD ACL Energy subscale scores for the Active Product versus Placebo comparison revealed no significant difference in the intention-to-treat (IT) analysis and the treatment received (TR) analysis. However, Global Energy Percent Change $(p=0.06)$ and Global Energy Scale Change $(p=0.09)$ both closely approached conventional levels of statistical significance for the active product in the IT analysis. Global Energy Percent Change $(p=0.05)$ and Global Energy Scale Change $(p=0.04)$ reached statistical significance in the TR analysis. A cumulative percent responders analysis graph indicated greater response rates for the active product.

Conclusions: OPC Factor ${ }^{\mathrm{TM}}$ may increase energy levels in healthy adults aged 45-65 years. A larger study is recommended. Clinical Trials.gov identifier: NCT03318019
\end{abstract}

\section{Introduction}

$\mathbf{P}$ eople report less overall energy in later life than at younger ages. ${ }^{1,2}$ Middle-aged people often note a decline in sustainable energy levels compared to when they were younger. Some food supplement manufacturers purport that their product increases energy levels. Many patients want to combine standard medical practices with complementary/alternative medicine (CAM) methods. ${ }^{3}$
Considering the prevalence of CAM use, it is important for physicians to have data from randomized controlled trials available to provide accurate information about CAM practices. ${ }^{1}$ Under the Dietary Health Supplement and Education Act, food supplement manufacturers and distributors can make statements about a product's effect on structure and function but not about the product's effects on diseases and disease symptoms without the same testing done for U.S. Food and Drug Administration (FDA)-approved phar-

${ }^{1}$ Penn-Presbyterian Medical Center, Philadelphia, PA.

${ }^{2}$ CCEB, University of Pennsylvania, Philadelphia, PA.

${ }^{3}$ Department of Biostatistics and Epidemiology, University of Pennsylvania School of Medicine, Philadelphia, PA.

${ }^{4}$ Department of Family Medicine and Community Health, University of Pennsylvania School of Medicine, Philadelphia, PA. 
maceuticals. We studied a food supplement, OPC Factor, ${ }^{\mathrm{TM}}$ (AlivenLabs, Lebanon, TN) to determine its effect on a normal function, namely, energy level variation.

In this study, we set out to test the efficacy of a product marketed with testimonials for increased energy. OPC Factor ${ }^{\mathrm{TM}}$ purportedly works by a synergistic combination of vitamins, minerals, and bioflavonoids called oligomeric proanthocyanidins (OPCs), which are powerful antioxidants 4,5 derived from grape seeds and pine bark. The effect of the product may be largely due to the antioxidant effects of oligomeric proanthocyanidins on aging mitochondria. ${ }^{6,7} \mathrm{Mi}-$ tochondria are the primary energy-producing components of cells. Moini et al. ${ }^{7}$ concluded that flavonoids reduce cytochrome $c$ and that pine bark extract inhibits electron transport chain activity in a study of rat liver mitochondria. Flavonoids exert their anti-oxidant activity through donating electrons.

No rigorous trial of the effect of OPCs or other antioxidants on energy levels in healthy persons is available. A few small clinical trials have evaluated the effect of nutritional supplements on fatigue secondary to specific pathology or of unknown etiology. Forsyth et al. ${ }^{8}$ performed a randomized, placebo-controlled, double-blind crossover study in 26 subjects with a stabilized form of NADH $\left(\mathrm{ENADH}^{\mathrm{TM}}\right)$ in chronic fatigue syndrome subjects. A significant difference in favor of the active product was found. Measurement relied on the clinical signs and symptoms. Another marketed nutritional product, NT Factor ${ }^{\mathrm{TM}}$ (a combination of phospholipids, fatty acids, vitamins, minerals, probiotics, and plant extracts) was tested alone or with another marketed vitamin/mineral product (Propax ${ }^{\mathrm{TM}}$; National Therapeutics, Inc., Haupage, NY) for chronic fatigue. Ellithorpe et al. ${ }^{9}$ reported a single-blinded pre-post design trial $(N=34)$ in which there was a $45.5 \%$ reduction in symptoms as assessed by the Piper Fatigue Scale, while Agadjanyan et al. ${ }^{10}$ reported in a blinded pretreatment, post-treatment, and postwashout study of 30 subjects a $35.5 \%$ reduction in fatigue scores on the Piper Fatigue Scale. The fatigue reduction scores correlated positively with a leukocyte mitochondrial function test. Cicero et al. ${ }^{11}$ reported a double-blinded, randomized, controlled trial in which 20 hypertensive subjects 65 years or older receiving digitalis were given either Siberian ginseng (Eleutherococcus senticosus maxim.) or placebo. Subjects taking Siberian ginseng had higher scores on the social functioning scale, mental health scale, and the mental component score of the 36Item Short Form Health Survey (SF-36) (a validated health status questionnaire ${ }^{12}$ ) after 4 weeks. However, the effect did not persist to 8 weeks. In summary, several small phase II studies showed promising results.

As in the studies reported above, we evaluated the study product as a whole. We did not attempt to determine the most active ingredient but to determine whether there was any effect on energy level for participants. Our study differs from other reports in several ways. First, we examined the effect of a nutritional supplement on normal variation in energy level and not on fatigue secondary to specific pathology. An extensive set of exclusion criteria was used to focus on normal energy variation. Second, we used a randomized, placebo-controlled, triple-blinded (investigators, subjects, and assessors were masked) crossover design to perform an efficacy evaluation.

\section{Materials and Methods}

\section{Study sample}

Subjects were recruited from the medical practices of the University of Pennsylvania Health System (UPHS). Recruitment techniques included flyers, advertisement in the campus newsletter, and letters sent to potential subjects who were identified in the UPHS database and who were prescreened by computer. All subjects were further screened by telephone and medical interview. This study was conducted according to U.S. and international standards of Good Clinical Practice (FDA Title 21 part 312 and International Conference on Harmonization guidelines), and applicable government regulations and institutional research policies and procedures. Protocols were reviewed and approved by the University of Pennsylvania Institutional Review Board (IRB). Permission was obtained from the potential subjects' physician before sending letters informing patients of the study. All participation was voluntary. Subjects signed IRB approved consent forms prior to participation.

\section{Eligibility criteria}

Eligible subjects were healthy individuals between the ages of 45 and 65 years, free of major medical and mental illnesses, which included AIDS, anemia, bipolar disease, cancer, chronic fatigue syndrome, congestive heart failure, chronic obstructive lung disease, depression, diabetes, drug/alcohol dependence, fibromyalgia, hypothyroidism, schizophrenia, and uncontrolled hypertension. To avoid energy variations associated with the menstrual cycle, only postmenopausal women were eligible. Medical conditions were ascertained by telephone interview using a checklist, chart review, and medical interview in combination. Eligible subjects had to have a score on the energy scale of the assessment instrument, described below, that indicated the possibility of at least $20 \%$ improvement.

\section{Measurement strategy}

Energy level. The primary energy measurement was the Energy subscale (scored 0-20) of the Activation Deactivation Adjective Check List (AD ACL), a validated instrument ${ }^{13-16}$ that has been used in a wide range of studies including drug and exercise studies. The AD ACL consists of a list of 20 adjectives. Subjects were instructed to rate each adjective in the context of how they felt at the moment they were making their responses. Participants were instructed to fill out the questionnaire at 7 PM on Friday or Saturday of each study week for 9 weeks.

Four single global measures ${ }^{17}$ of energy change and overall status change were used as secondary measures of outcome. Each domain (energy change and overall status change) was assessed in two ways (Table 1): one a measure of percent, the other a Likert scale (valued 1-7). In each method of assessment, subjects were instructed to make their responses in the context of "since this phase of the study began."

Other covariates. At the time of the screening interview, the intake data included age, sex, self-identified ethnicity, education level, global energy score, and the SF-36 questionnaire. ${ }^{12}$ The SF-36 is a validated general health status 
Table 1. The Four Single Global Change Questions

1. Since the start of this phase of the study my overall energy level has improved by:
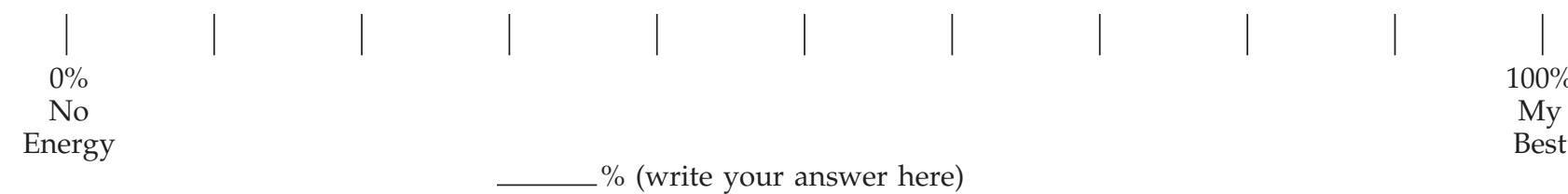

2. Since the start of this phase of the study my overall energy level is:

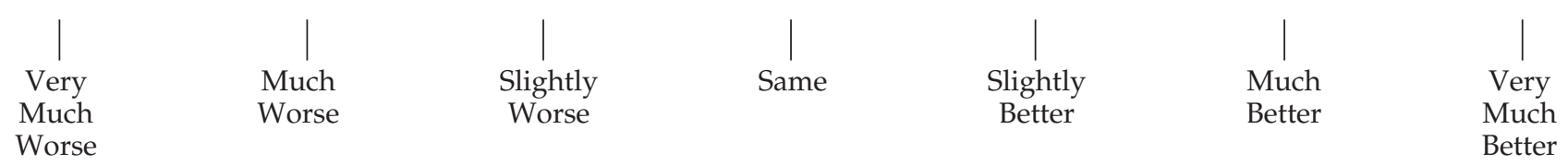

3. Since the start of this phase of the study my overall status has improved by:

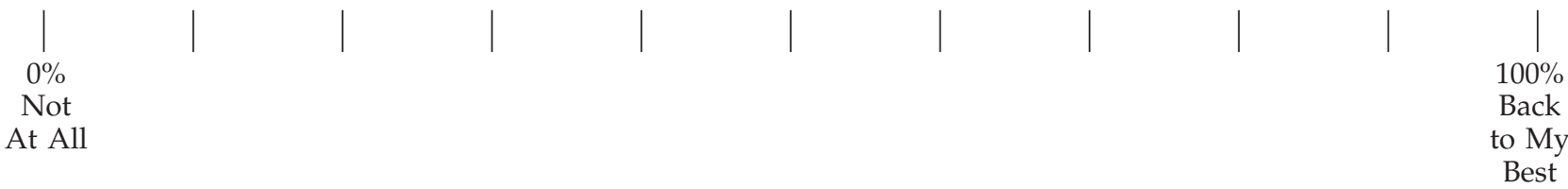

4. Since the start of this phase of the study my overall status is:

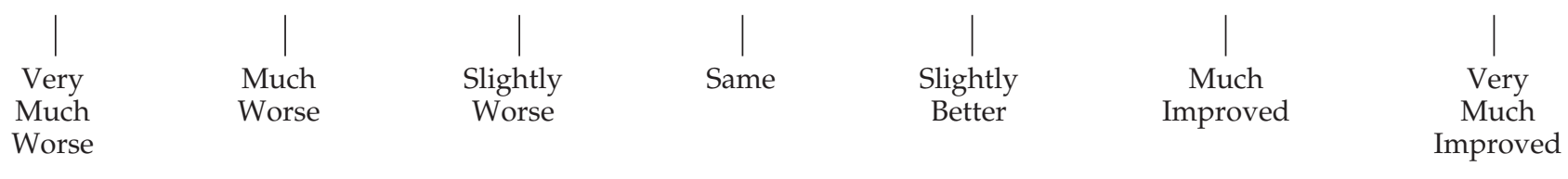

questionnaire consisting of eight subscales: Physical Functioning, Role-Physical, Bodily Pain, General Health, Vitality, Social Functioning, Role-Emotional, and Mental Health. Higher subscale scores indicate greater degrees of health. The SF-36 was administered once as an additional assessment of the health of the subjects participating in the study. It was not used as an outcome measure.

\section{Adherence and masking assessment}

Adherence was assessed by counting full and empty packets of the investigational product at the end of each phase. Masking success was assessed by comparison of the number of correct identifications of active product and placebo for subjects in the active product first sequence and the placebo first sequence of the first treatment phase. Subjects were asked to make an identification after the completion of each treatment phase of the study.

\section{Description of the product}

OPC Factor ${ }^{\mathrm{TM}}$ is in the form of a pink powder $(3.6 \mathrm{~g})$ that becomes effervescent in water due to an isotonic effervescent delivery system (glucose monomer, polymers, tartaric acid, potassium bicarbonate, magnesium oxide, malic acid, and citric acid). The product contains multiple vitamins and minerals along with grape seed and pine bark extract that furnishes OPCs. Maidenhair tree (Gingko biloba), bilberry, lycopene, lutein, and other nutritional components are also present. Laboratory and animal data are available on the safety and effects of OPCs, Gingko biloba, bilberry, and lycopene. ${ }^{18-44}$ Over the past 12 years, the individual ingredients in OPC Factor ${ }^{\mathrm{TM}}$ have been ingested by at least hundreds of thousands of people, while OPC Factor ${ }^{\mathrm{TM}}$ as a whole has been ingested by thousands of people over the last 5 years. The study product is a food supplement and thus is not regulated as a drug by the FDA. The placebo was an effervescent powder similar in appearance and taste but without nutrients.

Based on communication with the formulator of OPC Factor, ${ }^{\mathrm{TM}}$ consumers of the commercially available product ingest one to two packets per day. However, they may take three packets per day to get a response or as a loading mechanism. Subjects in our study ingested two packets per day, once in the morning and once in the evening.

\section{Study design}

The experimental design (Fig. 1) was a randomized, placebo-controlled, crossover design in which the investigators, subjects, and assessors were masked. The investigational pharmacy provided the randomization in blocks of size 2. Subjects were randomized to either placebo first or active product first. A 1-week baseline preceded the first phase and there was a 2-week washout period between the first phase and the second phase. Each phase was 3 weeks in duration. Assessments of change were made by subtracting the baseline score averages from phase 1 averages and washout score averages from phase 2 averages. The two dif- 


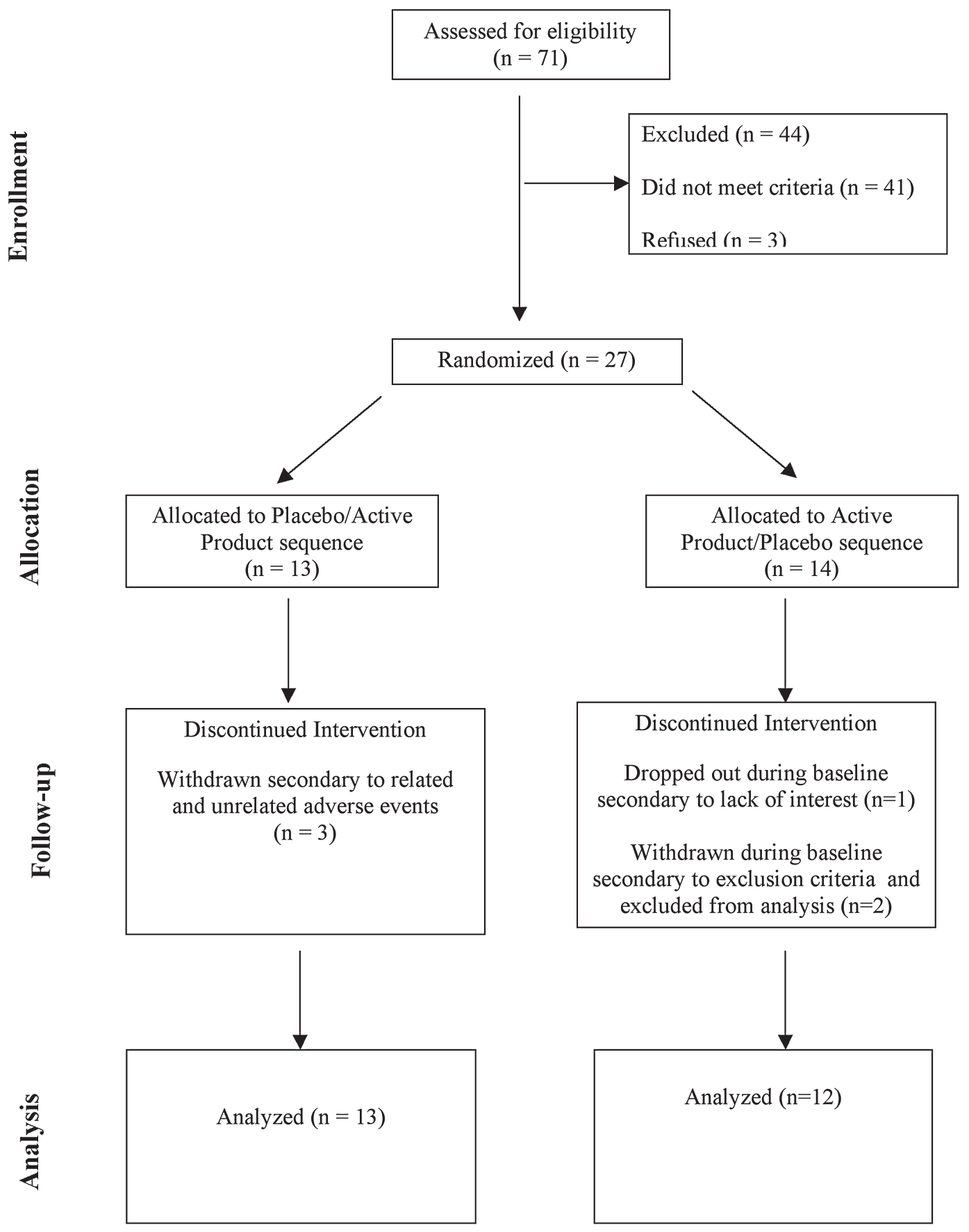

FIG. 1. Flow chart of progression through the study for the intention-to-treat analysis.

ferences for each subject (active and placebo) were then compared to each other.

\section{Analytic strategy}

The Energy subscale (AD ACL) endpoint was first examined for crossover/period effects by comparing the difference in AD ACL under active treatment and under placebo between the group randomized to the placebo first and the group randomized to the active product first. First the skewness / kurtosis tests were used to evaluate the distribution of each group. If either group was not normally distributed, the
Wilcoxon ranked-sum test was used for statistical testing. If both groups were normally distributed, a two sample $t$-test was used. If these analyses determined that there were no carryover/period effects, then the within-subject comparisons Placebo versus Active Product phases were conducted using either the Wilcoxon signed-rank test or paired $t$-test, depending on the normality of the distributions. The four single global change endpoints were evaluated similarly. Random effects linear regression techniques were used to adjust for baseline levels of energy and for the repeated measurements taken on the same subject over time in the Placebo versus Active Product analysis of the Energy subscale (AD 
ACL) endpoint. A cumulative proportion of responders analysis graph (CPRA) ${ }^{45}$ was constructed for the global endpoint of energy percent change. The masking analysis was performed by comparing the percent of correct identifications in each group (placebo first and active product first) using Fisher's Exact test. For all statistical tests, we employed a level of statistical significance set at $\alpha=0.05$, recognizing that tests of statistical significance are approximations that serve as aids to interpretation and inference. STATA version 9.2 (STATA Corp., College Station, TX) was used for all statistical analyses.

\section{Results}

\section{Sample characteristics}

The flow of the study for the intention-to-treat (IT) analysis is depicted in Figure 1. Of the 27 subjects randomized, 2 were withdrawn during the baseline period secondary to the detection of pre-existing exclusion criteria. The IT group was thus composed of 25 subjects. Of these 25 subjects, 1 subject dropped out during the baseline period and 3 were withdrawn secondary to study related and unrelated adverse events. The absence of these 4 subjects resulted in the treatment received (TR) group $(n=21)$.

The characteristics of the 25 subjects in the IT analysis group (Table 2) was as follows. The average age was 56 years. Males accounted for 17 (68\%) of the sample while females accounted for 8 (32\%). The racial mix indicated $21(84 \%)$ white; 2 (8\%) African-American; 1 (4\%) Hispanic; and 1 (4\%) Asian. Our sample reported a high level of educational attainment. Forty-eight percent (48\%) had at least a Master's degree. The screening score on the Energy subscale (AD ACL) averaged 10.28, while the screening global energy level was $68.75 \%$. The composition of the groups randomized to the placebo first sequence and the active product sequence were balanced on demographic factors. The SF-36 profile was at least as healthy as the national norm for the age group 55-64 years. Study group scores for the SF-36 were higher (healthier) on five of eight subscales with these exceptions: Role-Performance 73 versus 73.66; Vitality 58.33 versus 60.37; and Role-Emotional 79.71 versus 80.26. Compared to a reported sample with clinical depression, ${ }^{12}$ our sample scored higher on every subscale including Vitality 58.33 versus 40.12 and Role-Emotional 79.71 versus 38.90.

\section{Analysis of placebo/active product sequence versus active product/placebo sequence}

These data were viewed from two perspectives: Intention to Treat (IT) and Treatment Received (TR).

First, an analysis was conducted to determine the presence or absence of carryover or period effects associated with the two sequences (placebo/active and active/placebo) to which subjects were randomized. In both versions of analysis, there were no carryover/period effects. This was true for both the AD ACL and the four single global measures (Table 3).

\section{Analysis of active product versus placebo}

Given the results above, the next analysis examines subject improvement in energy when on active product compared to placebo (Table 4). There were no significant within subject differences in pre-post treatment change in energy level between placebo and active product as measured by the Energy subscale score of the AD ACL $(p=0.20)$. However, observed differences were in favor of the placebo. This reverse direction of association was present in both analytic strategies; IT and TR analyses approached conventional lev-

Table 2. Demographic and Clinical Characteristics of the IT Group $(N=25)$, All Subjects, Placebo/Active Group $(N=13)$, and Active/Placebo Group $(N=12)$ Prior to the Start of the Study

\begin{tabular}{lccc}
\hline & $\begin{array}{c}\text { All subjects } \\
\text { no. }(\%)\end{array}$ & $\begin{array}{c}\text { Placebo/active } \\
\text { no. }(\%)\end{array}$ & $\begin{array}{c}\text { Active/placebo } \\
\text { no. }(\%)\end{array}$ \\
\hline Age mean (SD), years & $56.7(5.5)$ & $54.7(5.5)$ & $58.9(4.8)$ \\
Gender & $17(68)$ & $8(61.5)$ & $9(75)$ \\
Male & $8(32)$ & $5(38.5)$ & $3(25)$ \\
Female & $1(4)$ & $0(0)$ & $1(8.3)$ \\
Race & $2(8)$ & $1(7.7)$ & $1(8.3)$ \\
Asian & $1(4)$ & $1(7.7)$ & $10(0)$ \\
African-American & $21(84)$ & $11(84.6)$ & $1(83.3)$ \\
Hispanic & $3(12)$ & $2(15.3)$ & $1(8.3)$ \\
White & $4(16)$ & $3(23.1)$ & $4(33.3)$ \\
Education level & $6(24)$ & $2(15.4)$ & $4(33.3)$ \\
High school or GED & $9(36)$ & $5(38.5)$ & $2(16.7)$ \\
Community college & $3(12)$ & $1(7.7)$ & $11(3.2)$ \\
Bachelor degree & $10.3(3.7)$ & $9.6(4.1)$ & $72.3(19.8)$ \\
Master/professional degree & $68.8(19.8)$ & $65.8(20.2)$ & \\
Doctoral/Post-Doctoral degree & & & \\
AD ACL mean (SD) & & & \\
Global energy level mean (SD), & & & \\
percent (N = 24) & & & \\
\hline
\end{tabular}

AD ACL, Activation-Deactivation Adjective Check List; IT, intention-to-treat analysis; GED, general equivalency diploma; SD, standard deviation.

${ }^{\mathrm{a}} N=24$ instead of 25 secondary to missing data for one subject. 
Table 3. Analysis for Carryover/Period Effects in the Group Receiving Active Product First $(N=14)$ Versus the Group Receiving Placebo First $(N=13)$

\begin{tabular}{|c|c|c|c|}
\hline & $\begin{array}{c}\text { Diff. means, }(S E), \\
(95 \% \text { CI })\end{array}$ & $\mathrm{p}$-value & Test statistic \\
\hline \multicolumn{4}{|l|}{ Intention to treat (IT) $N=25$} \\
\hline AD ACL & $0.85,(1.65)$ & 0.34 & Wilcoxon rank-sum \\
\hline Global Energy Percent Change & $-18.37(9.76)$ & 0.25 & Wilcoxon rank-sum \\
\hline Global Energy Scale Change & $-0.76,(0.52)$ & 0.30 & Wilcoxon rank-sum \\
\hline Global Overall Status Percent Change & $-14.37,[-34.54,5.81]$ & 0.15 & Two-sample $t$-test \\
\hline Global Overall Status Scale Change & $-0.61,(0.55)$ & 0.54 & Wilcoxon rank-sum \\
\hline \multicolumn{4}{|l|}{ Treatment received (TR) $N=21$} \\
\hline AD ACL & $-0.11,(1.75)$ & 0.72 & Wilcoxon rank-sum \\
\hline Global Energy Scale Change & $-20.75,(11.45)$ & 0.26 & Wilcoxon rank-sum \\
\hline Global Energy Scale Change & $-0.69,(0.63)$ & 0.62 & Wilcoxon rank-sum \\
\hline Global Overall Status Percent Change & $-16.27,[-43.13,10.59]$ & 0.21 & Two-sample $t$-test \\
\hline Global Overall Status Scale Change & $-0.51,(0.67)$ & 0.86 & Wilcoxon rank-sum \\
\hline
\end{tabular}

AD ACL, Activation-Deactivation Adjective Check List; CI, confidence interval; Diff. Means, difference of the means; SE, standard error of the difference of the means.

els of statistical significance. Analysis of covariance methods were also explored, which allow the observed change to vary depending upon the pretreatment baseline or washout period energy scores. The analysis was in favor of the placebo; however, the $p$-values were larger.

The analysis of the four global change questions is reported in Table 4. For these models, the direction of associations was consistent with the hypothesis of this study. In the IT analysis, Global Energy Percent Change $(p=0.06)$ and Global Energy Scale Change $(p=0.09)$ both closely approached conventional levels of statistical significance for the active product. In the TR analysis, Global Energy Percent Change $(p=0.05)$ and Global Energy Scale Change $(p=$ 0.04 ) both reached statistical significance, while Global Overall Status Scale Change $(p=0.11)$ changed in the direction of statistical significance. The IT analysis CPRA graph ${ }^{45}$ of global energy percent change demonstrated the proportion of responders for any chosen cut-off point (Fig. 2). The CPRA graph indicated that for any positive cut-off point, the active product had a higher proportion of responders than the placebo with the exception of one outlier point. At the $10 \%$ cut-off, the difference was $20 \%$ while at the $20 \%$ cut-off the difference was $12 \%$. In the TR analysis, the differences were $28.57 \%$ and $14.28 \%$, respectively.

\section{Adherence assessment}

The median adherence for the first phase of the study was $95.2 \%$ and $96.6 \%$ for the second phase.

\section{Masking assessment}

Comparison of the number of correct identifications in those subjects receiving active product first with those receiving placebo first indicated no significant statistical difference using Fisher's Exact test $(p>0.99)$. This analysis was based on the data available for 19 subjects of the IT group: 8 subjects in the active product first group and 11 subjects in the placebo first group.

\section{Adverse events}

The placebo was associated with one case of abdominal discomfort, and one case of a facial rash. The active product

Table 4. Analysis of Active Product Versus Placebo

\begin{tabular}{lccc}
\hline & $\begin{array}{c}\text { Difference in means, } \\
(S E),(95 \% \text { CI) }\end{array}$ & p-value & Statistical test \\
\hline Intention to treat (IT) $N=25$ & & & \\
AD ACL & $-1.06,(0.81)$ & 0.20 & Wilcoxon rank-sum \\
Global Energy Percent Change & $4.2,(5.13)$ & 0.06 & Wilcoxon rank-sum \\
Global Energy Scale Change & $0.18,(0.27)$ & 0.09 & Wilcoxon rank-sum \\
Global Overall Status Percent Change & $-1.95,(5.0)$ & 0.37 & Wilcoxon rank-sum \\
Global Overall Status Scale Change & $0.06,(0.28)$ & 0.06 & Wilcoxon rank-sum \\
Treatment received (TR) $N=21$ & $-1.69[-3.47,0.09]$ & 0.05 & Paired t-test \\
AD ACL & $5.12,(6.03)$ & 0.04 & Wilcoxon rank-sum \\
Global Energy Percent Change & $0.31,(0.31)$ & 0.32 & Wilcoxon rank-sum \\
Global Energy Percent Change & $-1.73,(5.93)$ & 0.11 & Wilcoxon rank-sum \\
Global Overall Status Percent Change & $0.17,(0.32)$ & Wilcoxon rank-sum \\
Global Overall Status Scale Change & & & \\
\hline
\end{tabular}

AD ACL, Activation-Deactivation Adjective Check List; CI, confidence interval; Diff. Means, difference of the means; SE, standard error of the difference of the means. 


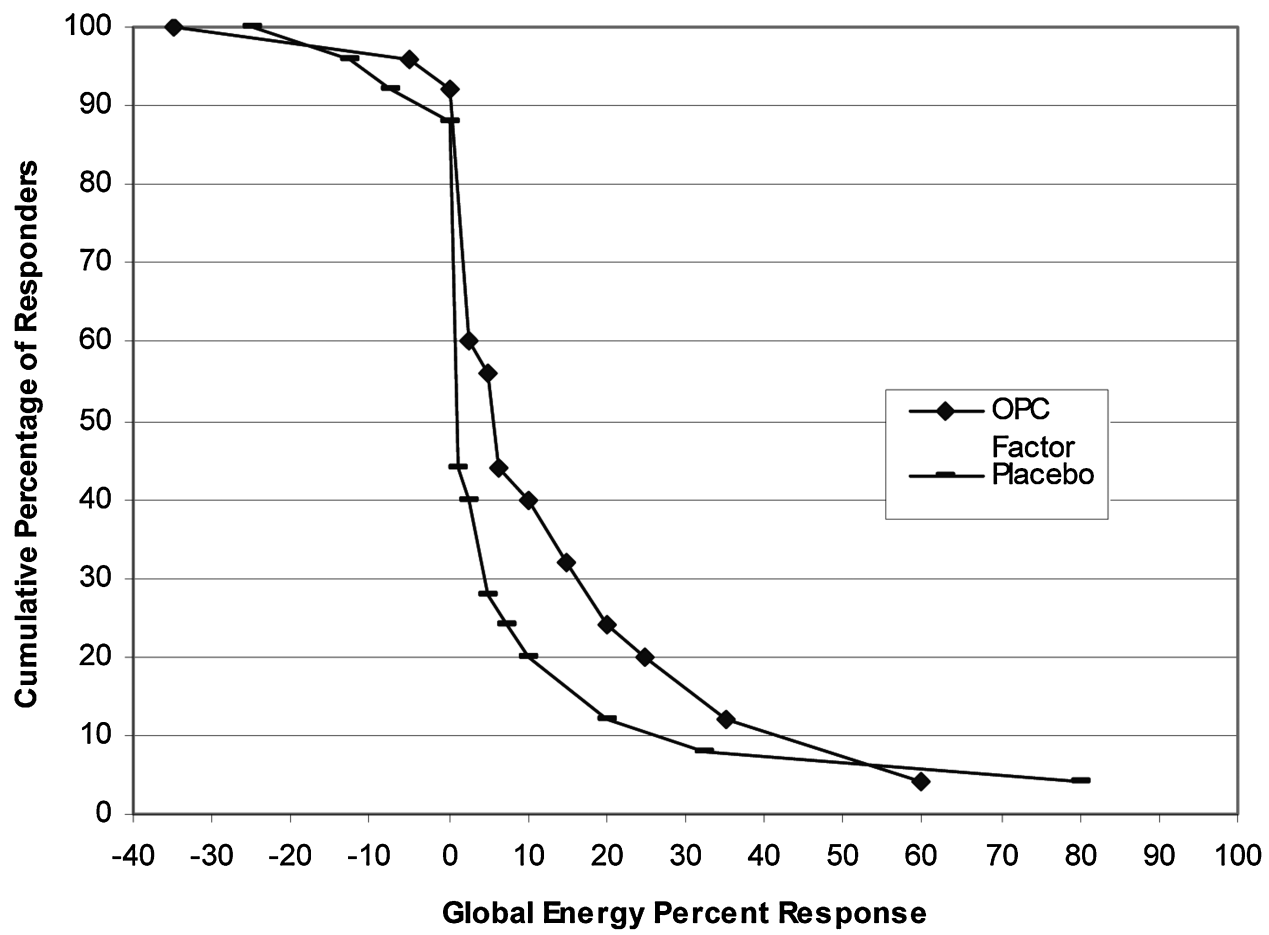

FIG. 2. Global Energy Percent Change/Cumulative Proportion of Responders Analysis Graph ( $N=25)$. For any chosen cutoff point on the horizontal axis, the percentage of responders with an equal or greater response is determined on the vertical axis.

was associated with one case of a rash on the legs and one case of a feeling of "jitteriness."

\section{Side benefits}

The active product was associated with one instance of improved eyesight in a subject who reported less dependence on reading glasses.

\section{Discussion}

This randomized, placebo-controlled, triple-blind crossover study employed the Energy subscale score of the AD ACL questionnaire as a primary outcome and four global change questions as secondary outcomes (Table 1). Two (2) of the global change questions related to energy status and two questions related to overall status. The AD ACL was answered in the time period context of "at this moment" while the global change questions were answered in the time period context of "since this phase of the study began" (Table 1). The AD ACL Energy subscale score showed no significant difference in placebo versus active product while the global change questions supported a positive effect of the active product compared to placebo in the context of a small phase II study.

Before putting our results in the context of other studies, the limitations should be discussed. The limitations relate to the sample size, the gender, ethnic and educational composition of our sample, and the employment strategy of the AD ACL. A larger sample size would give greater confidence in generalization of the results and increased power for the global change questions. We mitigated the disadvantages of a small sample by employing a crossover design to increase power. The sample size is appropriate for a phase II trial. The limitation of gender, ethnic, and educational composition is problematic in all small trials. However, this sampling problem is acceptable in the context of phase II studies that lead to larger studies. The third limitation is the employment strategy of the AD ACL. The AD ACL assesses moment-tomoment changes. This allows for greater sensitivity to change. However, this generates greater variability. Theoretically, this variability can be managed by averaging repeated measurements. We did average two of these weekly measurements in determining the Energy subscale scores for the placebo and active product phases. A better employment strategy would have been to have subjects complete the AD ACL daily and then average the daily scores. This was not possible secondary to funding issues. Three (3) subjects complained that the day they filled out the questionnaire did not always represent how they felt during the rest of the week. Subjects filled out questionnaires on Fridays or Saturdays at $7 \mathrm{PM}$. This corresponded to the day of the week on which the subject was randomized, which corresponded to the availability of clinical space. The Friday/Saturday problem was mitigated in the secondary outcome measures because the questions were answered in the time period context of "since this phase of the study began" (Table 1).Thus, subjects averaged their answers over the course of a week. Our conclusions focused on our secondary endpoints in light of the limitations of our primary outcome measure.

This randomized, placebo-controlled, crossover study supports the efficacy of the active product when measured by the single global questions for Energy Percent Change, 
Energy Scale Change, and Overall Status Scale Change questions. The use of single global questions and their relationship to recall has been reviewed. ${ }^{17,46,47}$ As a phase II study, the results clearly indicate that a larger study is in order. Our conclusions were supported by (1) The $p$-values in the IT analysis for Global Energy Percent Change $(p=0.06)$, and Global Energy Scale Change $(p=0.09)$ suggest statistical significance could be reached with a reasonably larger sample size ( $n=25$ in our study); (2) The TR analysis of sample size 21 reached statistical significance for Global Energy Percent Change $(p=0.05)$ and Global Energy Scale Change $(p=$ $0.04) ;(3)$ The TR analysis suggests a reasonably larger $n$ could result in Global Overall Status Scale Change $(p=0.11)$ reaching statistical significance; (4) In crossover designs, the randomization pertains to the between-groups analysis of carryover (sequence)/period effects (Fig. 1) while analysis of treatment effects is a within-group analysis in which each subject serves as their own control. Thus, exclusion of withdrawn subjects from the treatment effects analysis decreases generalizability of treatment results but does not jeopardize internal validity as it does in a parallel group design study. Thus, we utilize information from the TR analysis; and (5) The CPRA graph of Global Energy Percent Change for both the IT analysis (Fig. 2) and the TR analysis indicated that for any positive threshold, the active product had a higher proportion of responders than the placebo with the exception of one outlier.

This study differs from other related food supplement studies in that the focus was on healthy subjects and function instead of diseased subjects and a disease symptom (fatigue). Currently, there is no work available to define what a useful or clinically significant energy increase is for healthy subjects. Since we are in a new field of study, more time will be required before any consensus statement is made, such as the American College of Rheumatology's statement that a 20\% decrease in pain is considered clinically significant for patients with rheumatoid arthritis. ${ }^{48}$ The practical or clinical significance of the changes in the variation of energy levels of normal subjects can be better assessed with a large enough sample size that can be analyzed with parametric statistics rather than nonparametric statistics or that can be analyzed by comparing the proportion in each group that reaches a predetermined criterion of improvement. Our small sample size with non-normally distributed data makes interpretation of differences in means (Tables 3 and 4) difficult; therefore, most of our analyses were carried out with nonparametric statistical tests.

Our randomized, placebo-controlled, triple-blind crossover design has not been employed in other studies. Forsyth et al. ${ }^{8}$ used a randomized, placebo-controlled, double-blind design to study the effect of a stabilized form of NADH on subjects with chronic fatigue syndrome. Ellithorpe et al. ${ }^{9}$ and Agadjanyan et al. ${ }^{10}$ studied the effect of lipid replacement and antioxidants on chronic fatigue subjects. They used pre-post designs and incorporated standardized fatigue outcome measures. Cicero et al. ${ }^{11}$ studied the effect of a Siberian ginseng on SF-36 $6^{12}$ subscale scores of elderly hypertensive subjects on digitalis using a randomized, placebocontrolled, double-blind design.

Our study fits into the context of a beginning wave of phase II studies evaluating the effectiveness and efficacy of food supplements for enhancing structure and function (as described in the Dietary Health Supplement Education Act) and for the treatment of disease. Such studies help develop a research database of preliminary information, point to those supplements deserving of phase III trials, and inform the design of such studies.

This OPC Factor ${ }^{\mathrm{TM}}$ study points to the need for a larger phase III study and encourages investigators to take a closer look at other testimonials regarding this product such as improvement in visual acuity, macular degeneration, improved blood sugar control, and exercise performance. OPC Factor ${ }^{\mathrm{TM}}$ may enhance energy levels and overall health status in healthy adults aged 45-65 years. A larger and more balanced racial and socioeconomic sample is recommended. Qualitative studies could help define what a useful or clinically significant increase of energy is in healthy adults 45 and older. Our template can be the basis for larger future studies.

\section{Acknowledgments}

Dr. LaRiccia was supported by an NCCAM TR32 training grant. Small grants were provided by the Bach Fund of PennPresbyterian Medical Center and the Institute for Global Physiology and Behavior Research. Aliven Labs donated the study product and placebo but had no role in the analysis of the data or the reporting of the results. The work of Teresa Hogan, B.S., C.C.R.A., Sharon Hurley, B.A., Margaret Fuhs, R.N., Ph.D., Barbara Thompson, Clare Hayes, B.A., Gurneet M. Singh, R.Ac., N.D., Katherine Volk, B.A., Lic.Ac., Christina Palmer, B.A., Suzanne McMurphy, Ph.D., Sherry Morgan, M.L.S., R.N., and Sheila Sweeny, B.A., is gratefully appreciated. Grateful acknowledgement is also made to those physicians facilitating subject recruitment: Michael Baime, M.D., Michael Cirigliano, M.D., Carol Fleischman, M.D., Thorne Sparkman, M.D., and George Steele, M.D. Much appreciation is given to the staff of the Office of Human Research at the University of Pennsylvania School of Medicine: especially Greg Fromell, M.D., Mark Weiner, M.D., Marcia Markowitz, M.A., and Brette Brickel, M.A., for their guidance. The input of Robert Thayer, Ph.D. (author of the AD ACL) and Leann Bloeden, R.D., M.S. is appreciated.

This was an investigator sponsored study.

Perceived or potential conflicts of interest: During 2004, Dr. LaRiccia received less than $\$ 450.00$ in commissions in relation to the sale of OPC Factor. ${ }^{\mathrm{TM}}$

\section{References}

1. Allman BL, Rice CL. Neuromuscular fatigue and aging: Central and peripheral factors. Muscle Nerve 2002;25:785-796.

2. Trolongo P, Respini D, Ferrau F. Fatigue and aging. Crit Rev Oncol Hematol 2003;48(suppl):S57-S64.

3. Eisenberg DM, Davis RB, Ettner SL, et al. Trends in alternative medicine use in the United States, 1990-1997: Results of a follow-up national survey. JAMA 1998;280: 1569-1575.

4. Bagchi D, Bagchi M, Stoh SJ, et al. Free radicals and grape seed proanthocyanidin extract: Importance in human health and disease prevention. Toxicology 2000;148:187-197.

5. Nuttall SL, Kendall MJ, Bombardelli E, et al. An evaluation of the antioxidant activity of a standardized grape seed extract, Leucoselect. J Clin Pharm Ther 1998;25:385-389. 
6. Meissner C, Mohamed SA, von Wurmb N, et al. The mitochondria genome and aging. Z Gerontol Geriatr 2001; 34:447-451.

7. Moini H, Arvoyo A, Vaya J, et al. Bioflavonoid effects on the mitochondrial respiratory electron transport chain and cytochrome c redox state. Redox Rep 1999;4:35-41.

8. Forsyth LM, Preuss HG, MacDowell AL, et al. Therapeutic effects of oral NADH on the symptoms of patients with chronic fatigue. Ann Allergy Asthma Immunol 1999;82:185-191.

9. Ellithorpe RR, Settineri RA, Nicolson GL. Pilot study: Reduction of fatigue by use of a dietary supplement containing glycophospholipids. J Am Nutraceut Assoc 2003;6:23-28.

10. Agadjanyan M, Vasilevko V, Ghochikyan A, et al. Nutritional supplement (NT Factor ${ }^{\mathrm{TM}}$ ) restores mitochondrial function and reduces moderately severe fatigue in aged subjects. J Chron Fatigue 2003;11:23-36.

11. Cicero AF, Derosa G, Brillante R, et al. Effects of Siberian ginseng (Eleutherococcus senticosus maxim.) on elderly quality of life: A randomized clinical trial. Arch Gerontol Geriatr Suppl 2004;19:69-73.

12. Ware JE, Snow KK, et al. SF-36 Health Survey: Manual and Interpretation Guide. Lincoln, RI: QualityMetric Inc., 1993, 2000.

13. Thayer RE. Measurement of activation through self-report. Psychol Rep 1967;20:663-678.

14. Thayer RE. Factor analytic and reliability studies on the Activation-Deactivation Adjective Checklist. Psychol Rep 1978;42:747-756.

15. Thayer RE. Activation-Deactivation Adjective Check List (AD ACL): Current overview and structural analysis. Psychol Rep 1986;58:607-614.

16. Ekkekakis P, Hall EE, Petruzzello SJ. Evaluation of the circumplex structure of the Activation Adjective Check List before and after a short walk. Psychol Sport Exercise 2005; 6:83-101.

17. Sloan JA, Aronson N, Cappellari JC, et al. Assessing the clinical significance of single items relative to summated scores. Mayo Clin Proc 2002;77:479-487.

18. Oligomeric proanthocyanidins: Monograph. Altern Med Rev 2003;8:442-443.

19. Putter M, Grotemeyer KH, Wurthwein G, et al. Inhibition of smoking-induced platelet aggregation by aspirin and pycnogenol. Thromb Res 1999;95:155-161.

20. Yamakoshi J, Saito M, Kataoka S, et al. Safety evaluation of proanthocyanidin-rich extract from grape seeds. Food Chem Toxicol 2002;40:599-607.

21. Chang WC, Hsu FL. Inhibition of platelet aggregation and arachidonate metabolism in platelets by procyanidins. Prostaglandins Leukot Essent Fatty Acids 1989;38:181-188.

22. Pruess HG, Montomarry S, Echard B, et al. Long-term effects of chromium, grape seed extract, and zinc on various metabolic parameters of rats. Mol Cell Biochem 2001;223: 95-102.

23. Ray S, Bagchi D, Lim PM, et al. Acute and long-term safety evaluation of a novel IH636 grape seed proanthocyanidin extract. Res Commun Mol Pathol Pharmacol 2001;109:165-197.

24. Wren AF, Cleary M, Frantz C, et al. 90 Day oral toxicity study of a grape seed extract (IH636) in rats. J Agric Food Chem 2002;27;507:2180-2192.

25. Mizutani K, Ikeda K, Kawai Y, et al. Extract of wine phenolics improves aortic biomechanical properties in strokeprone spontaneously hypertensive rats (SHRSP). J Nutr Sci Vitaminol (Tokyo) 1999;45:95-106.
26. Nair N, Mahajan S, Chawda R, et al. Grape seed extract activates TH1 cells in vitro. Clin Diagn Lab Immmunol 2002; 9:470-476.

27. Bagchi D, Garg A, Krohn RL, et al. Protective effects of grape seed proanthocyanidins and selected antioxidants against TPA-induced hepatic and brain lipid peroxidation and CAN fragmentation, and peritoneal macrophage activation in mice. Gen Pharmacol 1998;30:771-776.

28. Banerjee B, Bagchi D. Beneficial effects of a novel IH636 grape seed proanthocyanidin extract in the treatment of chronic pancreatitis. Digestion 2001;63:203-206.

29. Avramovic V, Vlahovic P, Mihailovic D, et al. Protective effect of a bioflavonoid proanthocyanidin-BPI in glycerol-induced acute renal failure in the rat: Renal stereological study. Renal Fail 1999;21:627-634.

30. Foo LY, Lu Y, Howell AB, et al. A-type proanthocyanidin trimers from cranberry that inhibit adherence of uropathogenic p-fimbriated Escherichia coli. J Nat Prod 2000;63: 225-228.

31. Roychowdhary S, Wolf G, Keilhof G, et al. Protection of primary glial cells by grape seed proanthocyanidin extract against nitrosative/oxidative stress. Nitric Oxide 2001;5: 137-149.

32. Ambrogini $P$, Cuppini $R$, Bruno $C$, et al. Effects of proanthocyanidin on normal and reinnervated rat muscle. Boll Soc Ital Biol Sper 1995;71:227-234.

33. Tedesco I I, Russo M, Russo P, et al. Antioxidant effect of red wine polyphenols on red blood cells. J Inst Food Sci Technol 2000;11:114-119.

34. Meunier MT, Villie F, Bastide P. The interaction of Cupressus sempervirens L. proanthocyanidolic oligomers with elastase and elastins. J Pharm Belg 1994;49:453-461.

35. Takahashi T, Kamiya T, Yokoo Y, et al. Proanthocyanidins from grape seeds promote proliferation of mouse hair follicles in vitro and convert hair cycle in vivo. Acta Derm Venereol 1998;78:428-432.

36. Lininger SW, Gaby AR, Austin S, et al. The Natural Pharmacy. Roseville, CA: Prima Publishing, 1999:428-429.

37. Sierpina VS, Wollschlaeger B, Blumenthal M, et al. Gingko biloba. Am Fam Physician 2003;68:923-926.

38. Engelsen J, Nielsen JD, Winther K, et al. Effect of coenzyme $\mathrm{Q}_{10}$ and Gingko biloba on warfarin dosage in stable, longterm warfarin treated outpatients: A randomized, double blind, placebo-crossover trial. Thromb Haemost 2002;87: 1075-1076.

39. Lininger SW, Gaby AR, Austin S, et al. The Natural Pharmacy. Roseville, CA: Prima Publishing, 1999:396-397.

40. Abebe W. Herbal medication: Potential for adverse interactions with analgesic drugs. J Clin Pharm Ther 2002;27: 391-401.

41. Lininger SW, Gaby AR, Austin S, et al. The Natural Pharmacy. Roseville, CA: Prima Publishing, 1999:308-309.

42. Trumbo PR. Promises and perils of lycopene/tomato supplementation and cancer prevention-are there adverse effects of lycopene exposure? J Nutr 2005;135:2060S-2061S.

43. Lininger SW, Gaby AR, Austin S, et al. The Natural Pharmacy. Roseville, CA: Prima Publishing, 1999:308.

44. Alves-Rodrigues A, Shao A. The science behind lutein. Toxicol Lett 2004;150:57-83.

45. Farrar JT, Dworkin RH, Max MB. Use of the cumulative proportion of responders analysis graph to present pain data over a range of cut-off points: Making clinical trial data more understandable. J Pain Symptom Manage 2006;31:369-377. 
46. Von Korrf M, Jensen MP, Karoly P. Assessing global pain severity by self-report in clinical and health services research. Spine 2000;25:3140-3151.

47. Middel B, Stewart R, Bouma J, van Sonderen E, et al. How to validate clinically important change in health-related functional status: Is the magnitude of the effect size consistently related to magnitude of change as indicated by a global question rating? J Eval Clin Pract 2001;7:399-410.

48. Felson DT, Anderson JJ, Boers M, et al., American College of Rheumatology. Preliminary definition of improvement in rheumatoid arthritis [see comments]. Arthritis Rheum 1995;38:727-735.

\author{
Address reprint requests to: \\ Patrick J. LaRiccia, M.D. \\ 446 Kennerly Road \\ Springfield, PA 19104
}

E-mail: lariccip@mail.med.upenn.edu 